\title{
Negative expression of PTEN identifies high risk for lymphatic-related metastasis in human esophageal squamous cell carcinoma
}

\author{
ZHENGUO SUN $^{1}$, NA $\mathrm{JI}^{2}$, MINGMING BI ${ }^{1}$, ZHIPING ZHANG ${ }^{1}$, XIANGYAN LIU ${ }^{1}$ and ZHOU WANG ${ }^{1}$ \\ ${ }^{1}$ Department of Thoracic Surgery, Provincial Hospital Affiliated to Shandong University, Jinan, Shandong 250021, P.R. China; \\ ${ }^{2}$ Faculty of Health Science, Southampton University, Highfield Campus, Southampton SO171BJ, UK
}

Received January 22, 2015; Accepted April 2, 2015

DOI: $10.3892 / o r .2015 .3928$

\begin{abstract}
The poor prognosis of esophageal squamous cell carcinoma (ESCC) is mainly attributed to higher lymphatic-related metastatic ability. Whether the loss of expression of the phosphatase and tensin homolog deleted on chromosome 10 (PTEN) is associated with lymphatic-related metastasis needs elucidation. In the present study, we assessed the mRNA and protein level of PTEN in ESCC by qRT-PCR and immunohistochemistry. The results showed PTEN mRNA level in tumors was significantly lower than that in corresponding non-tumor esophageal epitheliums $(\mathrm{p}<0.001)$, while $38(51.4 \%)$ tumor samples were negative for expression of PTEN in ESCC tumors. Then the association between negative expression of PTEN and lymphatic-related metastasis (lymph node metastasis/3-year postoperative lymphatic metastatic recurrence) was evaluated. The proportion of PTEN-negative expression was significantly higher in positive lymph node metastasis $(\mathrm{pN}+)$ than that in negative lymph node metastasis (pN0) $(\mathrm{p}=0.021)$. The negative expression of PTEN was not an independent risk factor for the lymphatic recurrence rate in multivariate analysis $(\mathrm{p}=0.498)$, however, the lymphatic recurrence rate $(60.5 \%)$ in PTEN-negative expression group was higher than that (36.1\%) in PTEN-positive expression group $(\mathrm{p}=0.019)$. Furthermore, PTEN expression was stably silenced by lentiviral-vectored shRNA (Lenti-shRNA) in Eca109 (ESCC-derived cell line) to study functional effect of PTEN in vitro and in vivo. The laboratory study indicated increased cell proliferation, migration and invasion in vitro and more rapid growth rate of xenograft tumors in vivo after stable silencing of PTEN expression. Moreover, we proved that FAK/ pFAK were not the main factors mediating the mechanism of
\end{abstract}

Correspondence to: Professor Zhou Wang, Department of Thoracic Surgery, Provincial Hospital Affiliated to Shandong University, 324 Jingwu Road, Jinan, Shandong 250021, P.R. China E-mail: wz620226@hotmail.com

Key words: esophageal squamous cell carcinoma, metastasis, recurrence, PTEN, RNA interference, xenograft model metastasis in ESCC. In conclusion, negative expression of PTEN could be a useful biomarker to predict high risk for lymphatic-related metastasis in ESCC.

\section{Introduction}

Esophageal cancer is one of the most common cancers and the leading cause of cancer-related death worldwide. Esophageal squamous cell carcinoma (ESCC) is the predominant pathological type of esophageal cancer in China (1). Surgical resection remains the best choice for treatment of ESCC (2). However, the overall 5-year survival rate remains unsatisfactory with only $30-50 \%$ even after multimodal therapy (3). Lymphatic drainage of esophagus is fairly rich in mucoderm and muscularis mucosae, which lead to the higher incidence of lymphatic metastasis, comprising of lymph node metastasis and postoperative lymphatic metastatic recurrence. It is reported to be the main factor resulting in the poorer prognosis (4). Therefore, it is significant to identify molecular biomarkers that predict lymph node metastasis and postoperative lymphatic metastatic recurrence in ESCC.

The tumor suppressor gene, phosphatase and tensin homolog deleted on chromosome 10 (PTEN), also called TEP1 or MMAC1 and located at chromosome 10q23 region, is a dual lipid and protein phosphatase (5). Its primary function is to negatively regulate the phosphatidylinositol 3-kinase (PI3K)/AKT pathway, involving in the regulation of cell proliferation and apoptosis (6). PTEN was also reported to regulate cell migration and mobility in other mechanisms (7). PTEN activity is lost by mutation, deletions or promoter methylation silencing at high frequency in many human cancers (8). Some reports showed that loss of PTEN expression is associated with lymph node metastasis and poor prognosis in patients with gliomas, lung cancers, prostate and endometrial carcinomas, and gastric cancers (9-13). However, there are controversial results in whether loss of PTEN expression correlated with lymph node metastasis in ESCC (14-16). In addition, there is no previous report on whether loss of PTEN expression was associated with postoperative lymphatic metastatic recurrence in ESCC.

In the present study, we investigated the association between loss of PTEN expression and both lymph node 
metastasis and postoperative lymphatic metastatic recurrence in ESCC clinical samples. We further confirmed our clinical findings and investigated the possible mechanism mediating metastasis by lentivirus-mediated RNAi and assays in vitro and in vivo.

\section{Materials and methods}

Patient selection and follow-up. A total of 74 patients with mid-thoracic ESCC who underwent Ivor-Lewis esophagectomy with two-field lymph node dissection in our Department from January 2006 to December 2007 were enrolled in the present study. The informed consent was signed by all patients and the protocol was approved by the Ethics Committee of Provincial Hospital Affiliated to Shandong University. The inclusion criteria were as follows: i) complete tumor resection (R0). Cancer-free surgical and a lateral margins without residual foci were confirmed by pathological examination after surgery. Average lymph node number was $>12$. ii) All of the patients in the present study were re-staged according to 2009 UICC TNM staging for esophageal cancer. Those with stage I-III confirmed via pathological examination were included. iii) No preoperative radiotherapy or chemotherapy. iv) Those who died perioperatively were excluded.

Follow-up began on the date of surgery. All patients were examined routinely every 3 months and had detailed reports in the first 36 months after surgery. The patients underwent a comprehensive physical examination, chest and upper abdomen CT, abdominal B-ultrasonography or even PET-CT and biopsy at each checkup. Enlargement of lymph nodes in the cervical, supraclavicular, mediastinal or abdominal area was diagnosed as lymphatic metastatic recurrence. New lesions that were observed in other organs and excluded the primary tumor were diagnosed as hematogenous recurrence. The follow-up time was from 11 to 68 months and average follow-up time was 42.9 months.

RNA extraction and quantitative real-time PCR ( $q R T-P C R)$. Total RNA was extracted from fresh frozen tumor tissue, fresh corresponding non-tumor epitheliums and cell lines after transfection with TRIzol reagent (Invitrogen, USA) according to the manufacturer's instructions. The concentration and purity of RNA were determined by UV spectrophotometer with OD260/280 value between 1.8-2.0. Reverse transcription was performed using reverse transcriptase kit (Takara, Japan) in a volume of $20 \mu \mathrm{l}$, including $1 \mu \mathrm{g}$ RNA, dNTP, oligo(dT) primers, reverse transcriptase and reaction buffer. Then, qRT-PCR was performed in the LightCycler 480 (Roche, USA) by mixing $2 \mu \mathrm{l}$ cDNA with $7.2 \mu \mathrm{l} \mathrm{H}_{2} \mathrm{O}, 0.4 \mu \mathrm{l}$ forward and reverse primers, respectively, and $10 \mu \mathrm{l}$ SYBR-Green master mix (Takara) in a total volume of $20 \mu \mathrm{l} /$ reaction. The reaction conditions were $30 \mathrm{sec}$ at $95^{\circ} \mathrm{C}$ followed by 45 cycles of $30 \mathrm{sec}$ at $94^{\circ} \mathrm{C}, 30 \mathrm{sec}$ at $60^{\circ} \mathrm{C}$ and $30 \mathrm{sec}$ at $72^{\circ} \mathrm{C}$, and subsequently terminated at $4^{\circ} \mathrm{C}$ after 7 min elongation at $72^{\circ} \mathrm{C}$. The housekeeping gene GAPDH was used as an internal control for normalization of RNA quantity and quality. Gene-specific primers (Takara) are summarized as follows: PTEN (F, 5'-GA GCGTGCAGATAATGACAAGGAAT-3' and R, 5'-GGATTT GACGGCTCCTCTACTGTTT-3'); GAPDH(F, 5'-AGAAGGC
TGGGGCTCATTTG-3' and R, 5'-AGGGGCCATCCACAG TCTTC-3').

Immunohistochemistry. Immunohistochemical staining was performed on formalin-fixed paraffin embedded tumor tissues and corresponding non-tumor epithelium by the streptavidin peroxidase method. After deparaffinized, rehydrated and antigen retrieval, sections were incubated with $0.3 \%$ hydrogen peroxide for $30 \mathrm{~min}$ at $37^{\circ} \mathrm{C}$ to block endogenous peroxidase activity. After washing with phosphate-buffered saline (PBS) 3 times, sections were incubated with $1 \%$ bovine serum albumin for $30 \mathrm{~min}$ at $37^{\circ} \mathrm{C}$ to block non-specific reactions. Then sections were incubated overnight with monoclonal rabbit anti-human PTEN antibody at 1:50 dilution (Abcam, USA) at $4^{\circ} \mathrm{C}$. PBS was replaced as negative control. Subsequently, the secondary biotinylated antibody (Zhongshan Biotech, China) and avidin-biotin complex reagent were applied for $30 \mathrm{~min}$ at $37^{\circ} \mathrm{C}$, respectively. 3,3'-Diaminobenzidine tetrahydrochloride (Zhongshan Biotech) staining reaction was then performed. Sections were lightly counterstained with Mayer's hematoxylin and mounted.

All sections were examined by two independent pathologists who were blinded to the clinical data. The immunohistochemical score (IHS) was measured by combining the quantity score (percentage of positive stained cells) with the staining intensity score (16). The quantity score was observed as: 0 , if $<5 \%$; +1 , if $5-25 \%$; +2 , if $26-50 \%$; and +3 , if $>50 \%$ staining of tumor cells. The staining intensity was scored as 0 , absent; +1 , weak staining; +2 , moderate staining; and +3 , strong staining. The final score (range $0-9$ ) was the product of these two scores for each specimen. The total score was classified into negative expression/loss of expression (from 0 to 2 ) and positive expression (from 3 to 9).

Cell lines and cell culture. Human esophageal cancer cell lines Eca109, KYSE150, TE-1 and EC9706 were purchased from the Cell Bank of Shanghai Institute in China. RPMI-1640 medium with $10 \%$ fetal bovine serum (FBS) and $1 \%$ penicillin/streptomycin (both from HyClone, USA) were used for cell culture. Cells were grown in a humidified incubator containing $5 \%$ $\mathrm{CO}_{2}$ at $37^{\circ} \mathrm{C}$.

Western blotting. The protein of cells was extracted using RIPA lysis buffer plus phenylmethysulfonyl fluoride (PMSF) (Zhongshan Biotech) according to the manufacturer's instruction. After being on ice for $30 \mathrm{~min}$ and centrifugation for $30 \mathrm{~min}$, clear protein extracts were obtained from the supernatant. The protein concentration was determined by BCA protein assay kit (Pierce, USA) and the total protein was diluted in $5 \mathrm{X}$ loading buffer (Beyotime, China). Then $50 \mathrm{mg}$ of protein mixed with loading buffer was loaded/lane and separately by $10 \%$ SDS-polyacrylamide gel, with PageRuler Prestained Protein Ladder as size marker (Thermo Scientific, USA). Proteins were then transferred to polyvinylidene difluoride (PVDF) membranes (Millipore, USA). Membranes were incubated with TBS containing 0.1\% Tween-20 (TBST) and $5 \%$ non-fat dry milk for $1 \mathrm{~h}$ to block non-specific binding. The membranes were incubated overnight with monoclonal rabbit anti-human PTEN antibody at 1:500 dilution (Abcam), polyclonal rabbit anti-human FAK antibody at 1:500 or 
monoclonal rabbit anti-human p-FAK antibody at 1:500 (both from CST, USA) as well as polyclonal mouse anti-human $\beta$-actin antibody at $4^{\circ} \mathrm{C}$. Subsequently, the membranes were incubated with HRP-conjugated goat anti-rabbit or antimouse secondary antibodies (1:7,500) (Zhongshan Biotech) in TBST-5\% non-fat milk for $1 \mathrm{~h}$. Then immunoreactive bands were visualized using enhanced chemiluminescence (ECL) detection system (LAS-4000 mini system; GE, USA). Intensities of bands were measured by image analysis system (Imager Reader LAS-4000; GE) and normalized to $\beta$-actin as the endogenous control.

Lentivirus mediated short hairpin RNA (shRNA) knockdown of PTEN. By searching on GenBank, 3 target sequences and one negative control (NC) sequence were designed as follows: i) 5'-AGAGATCGTTAGCAGAAAC-3'; ii) 5'-GTATAGAGCGTGCAGATAA-3'; iii) 5'-AGAAC TTATCAAACCCTTT-3'; negative control, 5'-TTCTCCGAAC GTGTCACGT-3'. DNA oligos containing the sequences were chemically synthesized and annealed. Then they were inserted into pGCL-GFP vectors (GeneChem, China) by double digestion with AgeI and EcoRI and ligation with T4 DNA ligase (both from NEB, USA). Ligates were subsequently transformed into competent Escherichia coli DH5 cells and correct transformants were identified by colony PCR and DNA sequencing.

Lentivirus with shRNA1, 2 and 3 targeting PTEN (LentishRNA1, 2 and 3) and Lentivirus with negative control shRNA (Lenti-NC) were produced by co-transfection of ViraPower packaging mix (Invitrogen) and plasmids into $293 \mathrm{~T}$ cells according to the manufacturer's instruction. After $48 \mathrm{~h}$, the viral supernatant was harvest, passed through $0.45 \mu \mathrm{m}$ filters and concentrated. When viral titer was measured, Eca109 was transfected with lentivirus shRNAs, ENi.S (GeneChem) and $5 \mu \mathrm{g} / \mathrm{ml}$ Polybrene, followed by puromycin selection $(1 \mu \mathrm{g} / \mathrm{ml})$ for 10 days to obtain stably-transfected cells. Western blotting showed that Lenti-PTEN-shRNA2 has the highest inhibition rate, so that Eca109 transfected with Lenti-PTEN-shRNA2 was used in the following assays.

Cell proliferation assay. The proliferation of stably transfected cells was assessed with Cell Counting kit-8 (CCK-8) (Dojindo, Japan). Visuable cells $(3,000)$ per well were seeded in 96-well plates in a volume of $100 \mu \mathrm{l}$ (8-wells/group, total 5 plates). At the time of $24,48,72$ and $96 \mathrm{~h}$, a plate was added with $10 \mu \mathrm{lCCK}-8 /$ well and further incubated for $2 \mathrm{~h}$ at $37^{\circ} \mathrm{C}$ in $5 \% \mathrm{CO}_{2}$ to perform the assay, respectively. The absorbance at $450 \mathrm{~nm}$ was measured by a microplate reader. The experiment was performed in triplicate and the average of the results was analyzed.

Cell migration and invasion assay. For Transwell migration assay, cells were pre-cultured using serum-free medium for $24 \mathrm{~h}$. Cells $(30,000)$ in $200 \mu \mathrm{l}$ serum-free medium were added to upper chambers of 24-well Transwell apparatus (8- $\mu \mathrm{m}$ pore size) (Merck Millipore, Germany). The lower chambers were added with $500 \mu 1$ medium with $10 \%$ FBS. After incubation for $24 \mathrm{~h}$, cells remaining on the upper chambers were removed by scraping, while cells migrated through the membrane and attached to the underside were fixed in methanol and stained with hematoxylin and eosin. Then average cell numbers per visual field were counted under a light microscope (Leica DM 4000B; Leica, Germany).

For invasion assay, the upper chambers of the Transwell apparatus were pre-coated with $40 \mu$ l Matrigel (BD, USA) diluted 1:4 in serum-free medium and left to solidify for $1 \mathrm{~h}$. Then 50,000 cells pre-cultured in serum-free medium for $24 \mathrm{~h}$ were added to upper chambers in $200 \mu \mathrm{l}$ serum-free medium. Other procedures were the same to that of migration assay except that duration of incubation was $48 \mathrm{~h}$.

Xenograft tumor models in nude mice. A total of $15 \mathrm{BALB} / \mathrm{c}$ nude mice were purchased from the Vital River Company in Beijing. They were maintained in a temperature-controlled and pathogen-free animal facility with $12 \mathrm{~h}$ light/dark cycle at the Experimental Animal Centre of Provincial Hospital Affiliated to Shandong University. All mice were randomly divided into 3 groups: i) nude mice injected with Eca109 without transfection (Mock); ii) nude mice injected with Eca109 transfected with Lenti-NC; iii) nude mice injected with Eca109 transfected with Lenti-shRNA2. A total of $2 \times 10^{6}$ cells in $100 \mu \mathrm{l}$ were respectively injected into the right shoulder of mice. Tumor (xenograft) volumes were measured every week up to 6 weeks and calculated by the formula as previous study: $\mathrm{V}=\pi / 6 \mathrm{x}$ width ${ }^{2}\left(\mathrm{~mm}^{2}\right) \times$ length $(\mathrm{mm})(17)$. Mice were sacrificed and tumors were harvested after 6 weeks.

Statistical analysis. Paired t-test was used to compare the difference of PTEN mRNA in tumors and their corresponding non-tumor epithelia. Chi-square or Fisher's exact tests were used to study the differences of negative expression of PTEN in clinical characteristics. The association of clinicopathological characteristics and lymphatic metastatic recurrence was produced by log-rank test. Risk factors of postoperative lymphatic metastatic recurrence were analyzed with multivariate Cox regression analysis. Student's t-test was used to compare mean values in different groups. $\mathrm{P}<0.05$ was considered to indicate a statistically significant result. IBM SPSS version 19.0 was used for all the statistical analysis.

\section{Results}

Expression of PTEN mRNA in clinical samples. We examined PTEN mRNA level in 74 pairs of tumors and corresponding non-tumor esophageal epitheliums by performing qRT-PCR. The mean PTEN mRNA level $\left[(1.694 \pm 0.745) \times 10^{-2}\right]$ in tumor specimens was significantly lower than that $\left[\left(2.369 \pm 1.096 \times 10^{-2}\right]\right.$ in the corresponding non-tumor esophageal epithelia $(\mathrm{p}<0.001)$ (Fig. 1A).

Expression of PTEN protein in clinical samples and the correlation with lymph node metastasis ( $p N$ status). Expression of PTEN protein was analyzed in 74 tumor samples and 10 corresponding non-tumor esophageal epithelia by immunohistochemistry. It was shown that 38 (51.4\%) tumor samples were stained with negative expression of PTEN and $36(48.6 \%)$ tumor samples were stained with positive expression of PTEN. All the corresponding non-tumor esophageal epithelia were stained with positive expression of PTEN (Fig. 1B). The positive staining was mainly in cytoplasm and rarely nuclear (only 4 stained heterogeneously). 

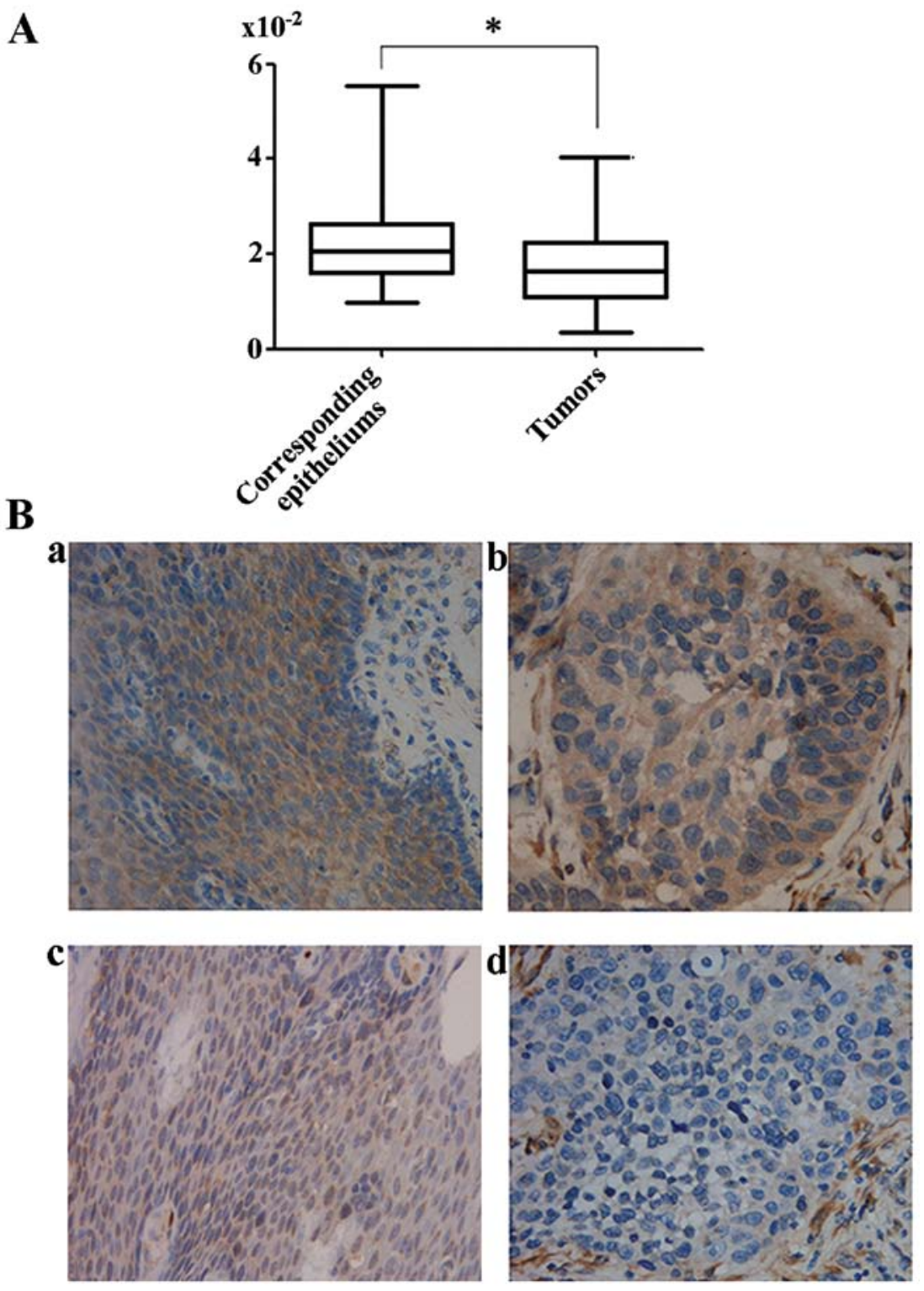

Figure 1. PTEN level by qRT-PCR and immunohistochemistry. (A) PTEN mRNA level in tumors was significantly lower than that in corresponding non-tumor esophageal epithelia. (B) PTEN protein expression by immunohistochemistry. a, positive expression in corresponding non-tumor esophageal epithelia; b, positive expression in cytoplasm in tumors; c, positive expression in nuclear in tumors; d, negative expression of PTEN in tumors. PTEN, phosphatase and tensin homolog deleted on chromosome 10.

Thirteen $(13 / 35,37.1 \%)$ tumors in pN0 group had negative expression of PTEN, while $26(25 / 39,66.7 \%)$ tumors in $\mathrm{pN}+$ group had negative expression of PTEN (Table I). Statistical analysis indicated that the proportion of negative expression of PTEN was significantly lower in pN0 than that in $\mathrm{pN}+$ $(\mathrm{p}=0.021)$. In addition, negative expression was also associated with $\mathrm{T}$ status, not with gender, age, loss of weight, tumor size and differentiation.

Negative expression of PTEN correlates with postoperative tumor metastatic recurrence. The overall 5-year survival rate in 74 ESCC patients was $31.1 \%$ (Fig. 2A). The detailed records of each patient in the first 36 months after surgery showed that 36 patients (48.6\%) experienced lymphatic metastatic recurrence, while 6 patients experienced hematogenous recurrence in other organs, such as liver, bone or lung. Patients (23 of 38) (60.5\%) with negative expression of PTEN experienced lymphatic metastatic recurrence, while 13 of 36 (36.1\%) patients with positive expression of PTEN experienced lymphatic metastatic recurrence (Fig. 2B). In univariate analysis, negative expression of PTEN was a significant factor for postoperative lymphatic metastatic recurrence $(\mathrm{p}=0.019)$, in addition to tumor depth (T status) and the presence of lymph node metastasis ( $\mathrm{N}$ status) (Table II). However, multivariate Cox regression showed that negative expression of PTEN was not an independent risk factor for lymphatic metastatic recurrence $(\mathrm{p}=0.498)$ (Table II).

Expression of PTEN in cell lines. We evaluated the expression of PTEN protein in four esophageal cancer cell lines: Eca109, KYSE150, TE-1 and EC9706 by performing western blotting. Western blot analysis showed that Eca109 had relatively high expression of PTEN (Fig. 3). Therefore, we selected Eca109 as the candidate cell line for shRNA transfection. 
Table I. Relationship between negative expression of PTEN and clinicopathological characteristics in 74 ESCC patients.

\begin{tabular}{|c|c|c|c|}
\hline $\begin{array}{l}\text { Clinicopathological } \\
\text { characteristics }\end{array}$ & $\begin{array}{l}\text { Patients } \\
(\mathrm{n}=74)\end{array}$ & $\begin{array}{l}\text { Negative expression } \\
\text { of PTEN }(\%)\end{array}$ & P-value \\
\hline Gender & & & 0.211 \\
\hline Male & 58 & $32(55.2)$ & \\
\hline Female & 16 & $6(37.5)$ & \\
\hline Age (years) & & & 1.000 \\
\hline$\geq 50$ & 64 & $33(51.6)$ & \\
\hline$<50$ & 10 & $5(50.0)$ & \\
\hline Loss of weight & & & 0.863 \\
\hline Yes & 15 & $8(53.3)$ & \\
\hline No & 59 & $30(50.8)$ & \\
\hline $\begin{array}{l}\text { Length of tumor } \\
(\mathrm{cm})\end{array}$ & & & 0.913 \\
\hline$<3$ & 17 & $8(47.1)$ & \\
\hline $3-5$ & 46 & $24(52.2)$ & \\
\hline$>5$ & 11 & $6(54.5)$ & \\
\hline T status & & & $0.005^{\mathrm{b}}$ \\
\hline $\mathrm{T} 1-2$ & 29 & $9(31.0)$ & \\
\hline $\mathrm{T} 3-4$ & 45 & $29(64.4)$ & \\
\hline $\mathrm{N}$ status & & & $0.021^{\mathrm{b}}$ \\
\hline N0 & 35 & $13(37.1)$ & \\
\hline N1-2 & 39 & $25(64.1)$ & \\
\hline Differentiation & & & 0.462 \\
\hline Well or moderate & 42 & $20(47.6)$ & \\
\hline Poor & 32 & $18(56.3)$ & \\
\hline
\end{tabular}

${ }^{\mathrm{a} C h i}$-square test or Fsher exact test; ${ }^{\mathrm{b}} \mathrm{p}<0.05$, ESCC esophageal squamous cell carcinoma; PTEN, phosphatase and tensin homolog deleted on chromosome 10 .

Transfection efficiency and selection of shRNA with higher RNAi efficiency in Ecal09 cell lines. After successful construction, Lenti-shRNA1, 2 and 3 and Lenti-NC were, respectively, transfected into Eca109. More than $90 \%$ of cells with GFP were considered as high efficiency of shRNA transfection for further detection and use. Western blotting showed that Eca109 transfected with Lenti-shRNA2 had the highest inhibition rate (Fig. 4). Thus, Eca109 transfected with LentishRNA2 and Eca109 transfected with Lenti-NC were chosen to perform the following assays in vitro and in vivo.

Stable knockdown of PTEN by Lenti-PTEN-shRNA2 increases cell proliferation. To evaluate the effect of PTEN on cell proliferation, we performed CCK- 8 assay and the cell growth curve was carried out according to the absorbance at detection. Eca109 transfected with Lenti-shRNA2 showed significantly increased cell growth rate compared with other groups (Fig. 5).

Stable knockdown of PTEN by Lenti-PTEN-shRNA2 increases cell migration and invasion. The effect of PTEN
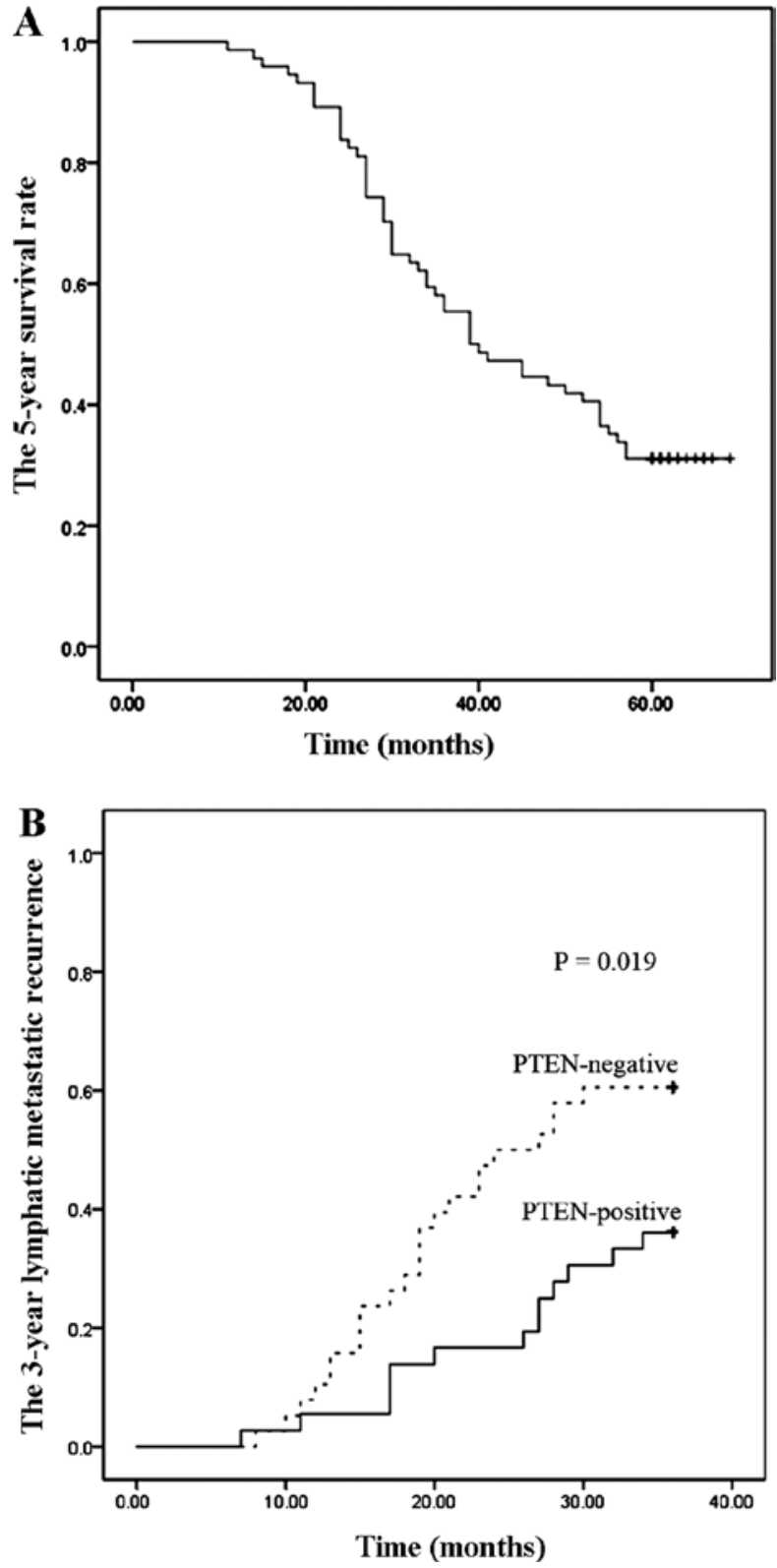

Figure 2. The prognosis of 74 ESCC patients. (A) The 5-year survival rate of ESCC patients. (B) The 3-year lymphatic metastatic recurrence in PTEN-negative group was significantly higher than that in PTEN-positive group. ESCC, esophageal squamous cell carcinoma; PTEN, phosphatase and tensin homolog deleted on chromosome 10.

on cell motility and invasion was tested using Transwell migration and invasion assays. The migration assay showed that Eca109 transfected with Lenti-shRNA2 had higher ability of migration than Eca109 with Lenti-NC (Fig. 6A and B). In the invasion assay, the invasive ability of Eca109 with Lenti-shRNA2 increased significantly compared with Eca109 with Lenti-NC (Fig. 6C and D). These results indicated that stable knockdown of PTEN may lead to the increase of metastatic ability in ESCC cells.

Stable knockdown of PTEN in xenograft tumor models in vivo. To further evaluate the effects of reduced PTEN expression on xenograft tumor growth in vivo, Eca109 cells mock treated, transfected with Lenti-NC or Lenti-shRNA2 were, respectively, injected into nude mice. The volume of subcutaneous 
Table II. Univariate and multivariate analysis of risk factors in 74 ESCC with respect to lymphatic metastatic recurrence in the first 3 years after surgery.

\begin{tabular}{lcccc}
\hline & \multirow{2}{*}{$\begin{array}{c}\text { Univariate } \\
\text { analysis } \\
\text { Risk factors }\end{array}$} & \multicolumn{3}{c}{ Multivariate analysis } \\
\cline { 3 - 5 } & P-value & P-value & Hazard ratio & $95 \%$ CI \\
\hline Gender & 0.374 & 0.899 & 1.065 & $0.403-2.813$ \\
Age (years) & 0.194 & 0.189 & 2.231 & $0.674-7.381$ \\
Length of tumor & 0.506 & 0.203 & 1.541 & $0.792-2.999$ \\
Loss of weight & 0.462 & 0.596 & 0.768 & $0.289-2.040$ \\
T status & $0.002^{\mathrm{a}}$ & $0.024^{\mathrm{a}}$ & 2.839 & $1.148-7.021$ \\
N status & $0.005^{\mathrm{a}}$ & $0.039^{\mathrm{a}}$ & 2.239 & $1.040-4.816$ \\
Differentiation & 0.229 & 0.684 & 0.857 & $0.408-1.802$ \\
Negative expression of PTEN (IHC) & $0.019^{\mathrm{a}}$ & 0.498 & 1.308 & $0.602-2.841$ \\
\hline
\end{tabular}

${ }^{\mathrm{a}} \mathrm{p}<0.05$; ESCC, esophageal squamous cell carcinoma; IHC, immunohistochemistry; CI, confidence interval; PTEN, phosphatase and tensin homolog deleted on chromosome 10.

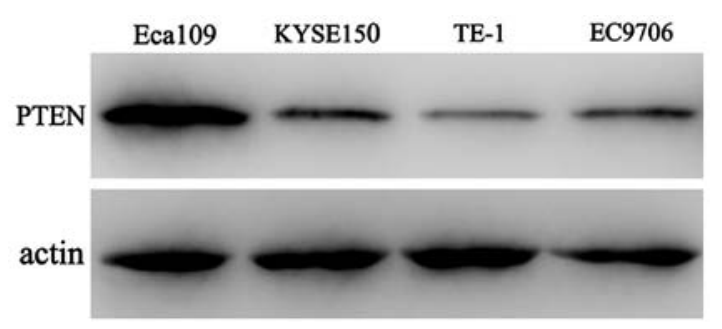

Figure 3. Protein expression of PTEN in different esophageal cancer cell lines. Eca109 showed higher expression of PTEN, thus it was chosen as the candidate cell line for shRNA suppression. PTEN, phosphatase and tensin homolog deleted on chromosome 10 .

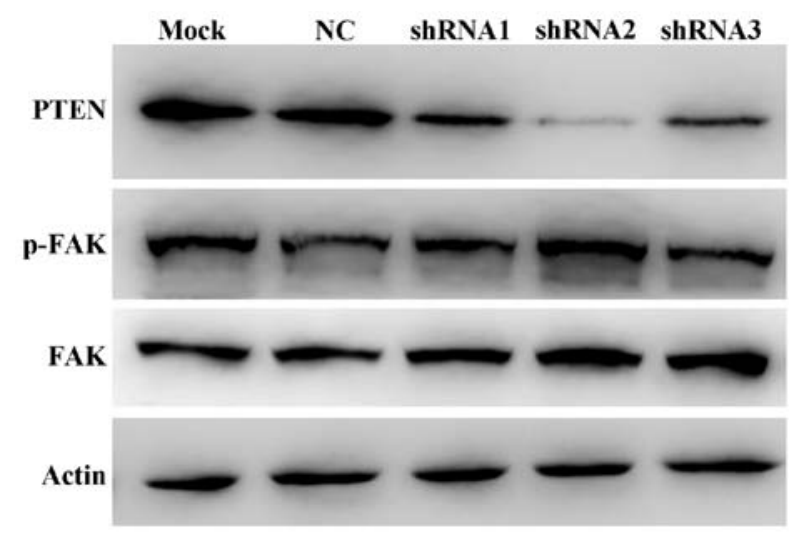

Figure 4. Suppression of PTEN gene expression in Eca109 by lentiviralvector shRNAs in western blotting and the mechanism mediating metastasis. shRNA2 showed strongest suppressing effect on PTEN and was selected for the following assays. Expression of FAK/pFAK did not have obvious change after suppression of PTEN gene expression, which revealed FAK/pFAK were not key factors mediating PTEN-related metastasis in ESCC. PTEN, phosphatase and tensin homolog deleted on chromosome 10; ESCC, esophageal squamous cell carcinoma.

tumors in Lenti-PTEN-shRNA2 group was shown to grow more rapidly than that in the other two groups (Fig. 7). The difference was statistically significant.

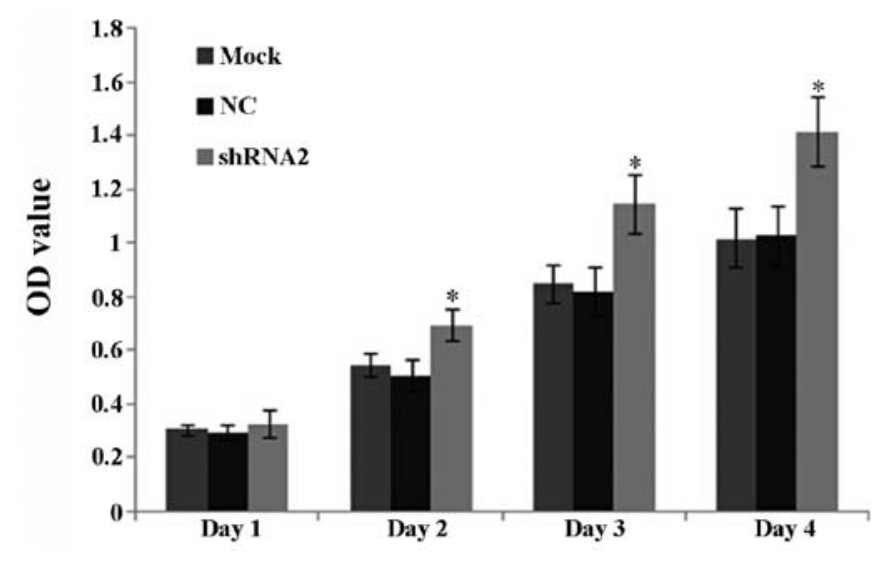

Figure 5. Effect of PTEN knockdown on cell proliferation. Cell proliferation was measured by CCK- 8 and represents 3 independent experiments. Cells transfected with Lenti-shRNA2 grew more rapidly than cells in the other two groups from the second day. PTEN, phosphatase and tensin homolog deleted on chromosome 10.

Study of possible mechanism in metastasis. Both results of clinical tissues and experimental cell lines in vitro and in vivo showed that negative expression of PTEN was correlated with metastasis. We further investigated the possible mechanism in the process of metastasis. We evaluated the changes of protein expression of FAK, p-FAK in Eca109 mock treated, with Lenti-NC, Lenti-shRNA1, 2 and 3 by western blotting. However, no remarkable changes in the expression were observed (Fig. 4). Therefore, possibly some other molecular mechanisms rather than FAK/p-FAK pathway mediate tumor metastasis in ESCC.

\section{Discussion}

Although loss of PTEN expression has been studied to correlate with lymph node metastasis in other tumors $(11,12)$, the function of PTEN in esophageal squamous cell carcinoma (ESCC) is still debated (14-16). Ding et al reported that there 

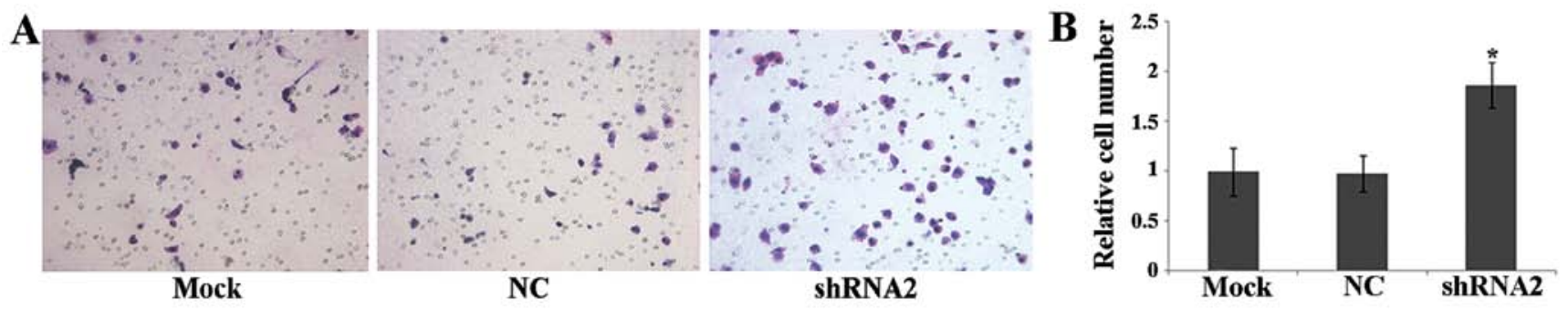

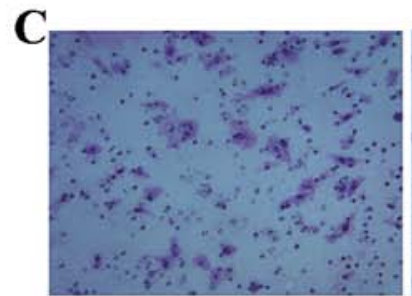

Mock

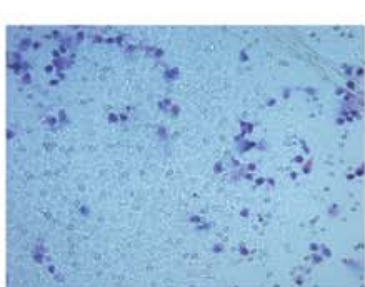

NC

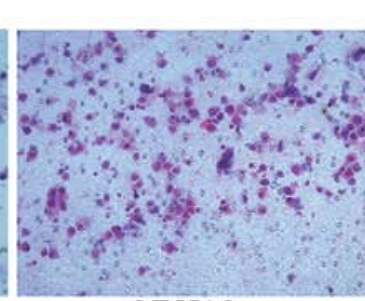

ShRNA2

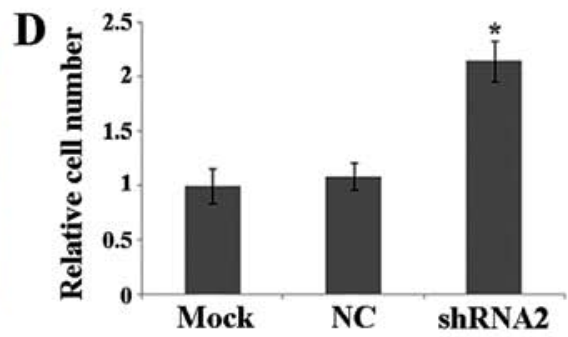

Figure 6. Effect of PTEN knockdown on cell migration and invasion. (A and B) Cells $(30,000)$ were added to upper chamber of 24-well plates and stained after $24 \mathrm{~h}$ culture in Transwell migration assay. The group transfected with Lenti-shRNA2 had the greatest cell number (stronger ability of migration) compared to the other two groups, in 3 independent experiments. (C and D) Cells $(50,000)$ were applied to the upper chamber of 24 -well plates and stained after $48 \mathrm{~h}$ culture in Transwell invasion assay. The average invasion cell counts in Lenti-shRNA2 group was more than that in the other two groups, indicating stronger invasive ability, in 3 independent experiments. PTEN, phosphatase and tensin homolog deleted on chromosome 10.
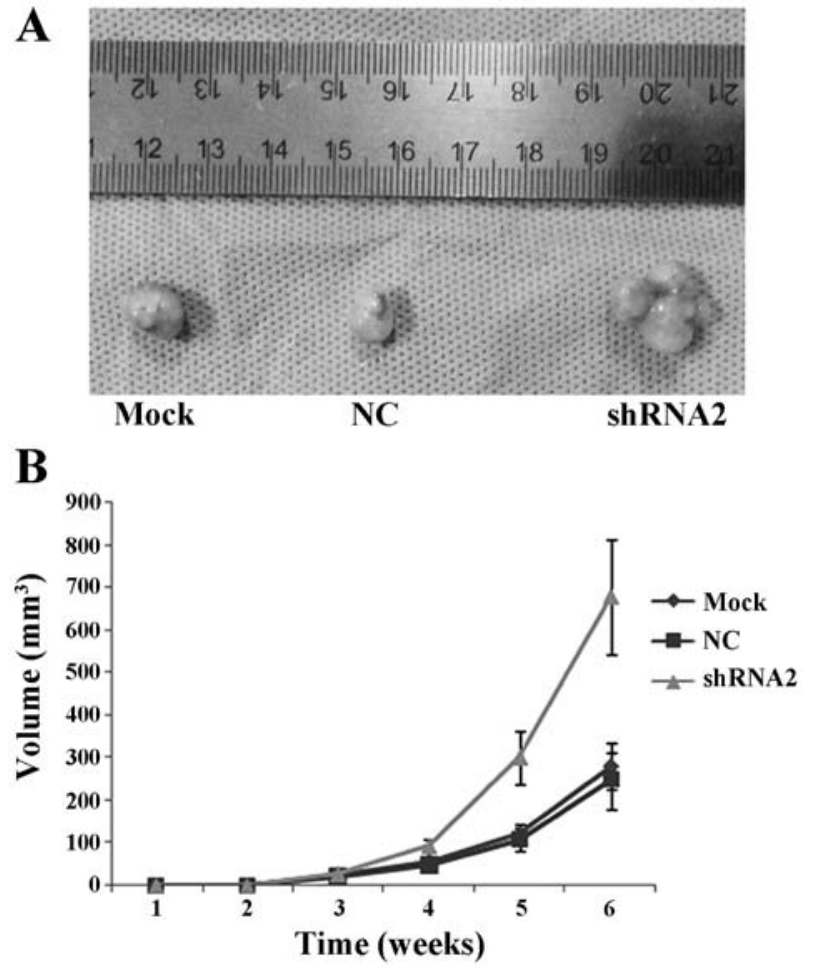

Figure 7. Effect of PTEN knockdown on xenograft tumors. (A) Eca109 untreated, transfected with Lenti-NC or Lenti-shRNA2 were, respectively, subcutaneously injected into nude mice. Representative image of xenograft tumors dissected after 6 weeks in 3 groups. (B) Volume of xenograft tumors were measured from 3 to 6 weeks. Every group included 5 nude mice, and statistical analysis showed that xenograft tumors grew more rapidly in Lenti-shRNA2 group, compared with Mock or Lenti-NC groups. PTEN, phosphatase and tensin homolog deleted on chromosome 10.

were no differences in protein and mRNA level between ESCC and corresponding normal esophageal epithelia (14). However, Tachibana et al (15) and Chang et al (16) showed that negative expression of PTEN in nuclear/cytoplasm correlated with tumor progression. In the present study, we proved the existence of negative expression of PTEN in mRNA and protein levels in ESCC tumors. We found that the mean PTEN mRNA level was significantly lower in tumors than that in corresponding non-tumor epithelia by qRT-PCR, while 38 (51.4\%) tumor samples were stained with negative expression of PTEN by IHC. This result was consistent with the findings of Tachibana et al (15) and Chang et al (16). Nevertheless, we proved that positive expression of PTEN was mainly located in the cytoplasm, only 4 had nuclear staining. This is inconsistent with Tachibana et al who reported a larger proportion of tumors with positive nuclear expression of PTEN (15). In our opinion, the different reports can be explained by use of different antibodies and evaluation threshold, or enrollment of patients from different areas. Further multicenter larger sample may be necessary to draw more convincing conclusions on expression of PTEN in ESCC.

ESCC has poor prognosis with strong metastatic ability related to rich lymphatic drainage in the esophagus, comprising of high lymph node and lymphatic metastatic recurrence after surgery $(18,19)$. Whether negative expression of PTEN is associated with this metastatic ability was not previously elucidated. Consistent with other tumors, we found that the proportion of negative expression of PTEN (64.1\%) in positive lymph node metastasis group $(\mathrm{pN}+)$ was significantly higher than that $(37.1 \%)$ in negative lymph node metastasis group (pN0). On the contrary, Chang et al reported the lymph node metastasis rate in negative expression of PTEN group $(7 / 16,43.8 \%)$ was higher than that in positive expression group $(13 / 48,14.6 \%)$ without statistical significance, revealing no obvious association between negative expression of PTEN and $\mathrm{N}$ status (16). Of note, according to previous studies in other tumors, negative expression was mainly observed in advanced tumors $(10,12)$, while a greater number of early ESCC patients were enrolled in the study of Chang et al (16). 
Subsequently, we firstly assessed the association between negative expression of PTEN and 3-year postoperative lymphatic metastatic recurrence in the follow-up. The negative expression of PTEN was not an independent risk factor for postoperative lymphatic metastatic recurrence in multivariate model, whereas, univariate analysis showed that the 3-year lymphatic metastatic recurrence rate $(60.5 \%)$ in PTEN-negative expression group was significantly higher than that $(36.1 \%)$ in PTEN-positive expression. Based on the above clinical analysis, we can draw the conclusion that negative expression of PTEN was correlated with lymphatic drainage-related metastasis, including lymph node metastasis (N stage) and lymphatic metastatic recurrence after surgery. To further prove our clinical findings and identify the mechanisms. We used shRNA to silence the expression of PTEN in ESCC cell lines and studied the effect of knockdown of PTEN on cellular ability related to tumor metastasis in vivo and in vitro.

RNA interference (RNAi) using small inhibitory RNA (siRNA) is a powerful method to downregulate the expression of mRNA and study function of target proteins $(20,21)$. However, synthesized siRNA only lasts for a short time, not allowing for stable suppression of the target gene. The lentiviral vectors, considered as ideal tool, can efficiently transduce exogenous siRNA with a stable suppression of target gene in vivo and in vitro $(22,23)$. Therefore, we chose lentiviral vectors for shRNA expression in our study. Eca109 was used to target suppression by shRNA due to its higher expression of PTEN. After confirmation of stable transfection, we proved Lenti-shRNA2 had a stronger suppressing effect on PTEN than the other two shRNAs, thus used to perform in vitro and in vivo assays.

Tumor metastasis is considered a multiple process involving the ability of primary tumor cells invading into the surrounding tissues and the ability of propagation at distal sites (24). Cell migration/invasion assay representing motility/invasive ability and cell proliferation assay representing propagation were performed to investigate the effect on tumor metastasis caused by Lenti-shRNA2 in vitro. The CCK- 8 assay showed Lenti-shRNA2, compared with Lenti-NC or mock, effectively increased the growth rates of Eca109. Migration and invasion assays confirmed that Eca109 knockdown of PTEN by Lenti-shRNA2 had stronger ability of motility and invasion than Eca109 with Lenti-NC or mock. We concluded knockdown of PTEN by Lenti-shRNA2 leads to increased ability of metastasis in vitro. To further validate the effect of silencing PTEN, we performed in vivo investigation in nude mice. The average volume of xenografts in Lenti-shRNA2 group was shown to be significantly larger than that in Lenti-NC or mock group. The in vivo study revealed that silencing PTEN resulted in increased tumorigenicity and tumor growth of xenografts, which was in accordance with the results in vitro. Both in vitro and in vivo studies confirmed our clinical finding that loss of PTEN was correlated with metastasis in ESCC.

Although loss of PTEN expression was associated with metastasis (25-27), the exact mechanism of PTEN in metastasis was still elusive. Early studies showed that PTEN interacted with the focal adhesion kinase (FAK) and reduced its tyrosine phosphorylation (p-FAK) to mediate cell spreading and migration $(7,28)$. Zhang et al reported that over- expression or knockdown of PTEN in gastric cancer resulted in downregulation or upregulation of FAK and decreased or increased cellular invasion through $\mathrm{PI} 3 \mathrm{~K} / \mathrm{NF}-\kappa \mathrm{B}$ pathway (29). Overexpression of PTEN in osteosarcoma cells downregulated the expression of FAK, p-FAK and matrix metalloproteinase-9 (MMP-9) (30). However, the western blotting in the present study showed that there were no remarkable differences of FAK and p-FAK among Eca109 cells mock or transfected with Lenti-NC, Lenti-PTEN-shRNA1, 2 and 3. We believed that there may be some other mechanisms contributing to the regulation of migration and invasion in ESCC cell lines, and further investigation in the mechanism are required.

In conclusion, negative expression of PTEN was closely associated with lymphatic-related metastasis, including lymph node metastasis $(\mathrm{pN})$ and a 3-year lymphatic metastatic recurrence after surgery in clinical analysis of ESCC. The laboratory experiments showed that silence of PTEN led to increased cell proliferation and migration, and invasion in vitro, and more rapid growth rate of xenografts tumor models in vivo, which validated our clinical findings. Negative expression of PTEN may be considered as a valuable tool to predict the possibility of lymphatic-related ESCC metastasis in future and to predict poor prognosis. The limitation of the present study is the small number of patients and lack of regular 5-year lymphatic metastatic recurrence data in detail. The prospective study of larger multicenter cases with longer follow-up data in detail may draw a more convincing conclusion.

\section{Acknowledgements}

The present study is supported by grants from the National Natural Science Foundation of China (no. 81172161), and the Shandong Provincial Science and Technology Development Plan (2012GSF11816). All authors are grateful to Professor Yu Liu, Department of Pathology, Provincial Hospital Affiliated to Shandong University for the assistance in specimens collection and staining evaluation.

\section{References}

1. Kamangar F, Dores GM and Anderson WF: Patterns of cancer incidence, mortality, and prevalence across five continents: Defining priorities to reduce cancer disparities in different geographic regions of the world. J Clin Oncol 24: 2137-2150, 2006.

2. Law S and Wong J: Two-field dissection is enough for esophageal cancer. Dis Esophagus 14: 98-103, 2001.

3. Song Y, Wang Z, Liu X, Jiang W and Shi M: CCR7 and VEGF-C: Molecular indicator of lymphatic metastatic recurrence in $\mathrm{pN} 0$ esophageal squamous cell carcinoma after Ivor-Lewis esophagectomy? Ann Surg Oncol 19: 3606-3612, 2012.

4. Chen G, Wang Z, Liu XY and Liu FY: Recurrence pattern of squamous cell carcinoma in the middle thoracic esophagus after modified Ivor-Lewis esophagectomy. World J Surg 31: 1107-1114, 2007.

5. MaehamaT and Dixon JE: The tumor suppressor,PTEN/MMAC1, dephosphorylates the lipid second messenger, phosphatidylinositol 3,4,5-trisphosphate. J Biol Chem 273: 13375-13378, 1998.

6. Shi Y, Paluch BE, Wang X and Jiang X: PTEN at a glance. J Cell Sci 125: 4687-4692, 2012.

7. Tamura M, Gu J, Takino T and Yamada KM: Tumor suppressor PTEN inhibition of cell invasion, migration, and growth: Differential involvement of focal adhesion kinase and $\mathrm{p} 130^{\mathrm{Cas}}$ Cancer Res 59: 442-449, 1999.

8. Blanco-Aparicio C, Renner O, Leal JF and Carnero A: PTEN, more than the AKT pathway. Carcinogenesis 28: 1379-1386, 2007. 
9. Kondo Y,Hollingsworth EF and Kondo S: Molecular targeting for malignant gliomas (Review). Int J Oncol 24: 1101-1109, 2004.

10. Tang JM, He QY, Guo RX and Chang XJ: Phosphorylated Akt overexpression and loss of PTEN expression in non-small cell lung cancer confers poor prognosis. Lung Cancer 51: 181-191, 2006.

11. Terakawa N, Kanamori Y and Yoshida S: Loss of PTEN expression followed by Akt phosphorylation is a poor prognostic factor for patients with endometrial cancer. Endocr Relat Cancer 10 203-208, 2003

12. Koksal IT, Dirice E, Yasar D, Sanlioglu AD, Ciftcioglu A, Gulkesen KH, Ozes NO, Baykara M, Luleci G and Sanlioglu S: The assessment of PTEN tumor suppressor gene in combination with Gleason scoring and serum PSA to evaluate progression of prostate carcinoma. Urol Oncol 22: 307-312, 2004

13. Im SA, Lee KE, Nam E, Kim DY, Lee JH, Han HS, Seoh JY, Park HY, Cho MS, Han WS, et al: Potential prognostic significance of p185(HER2) overexpression with loss of PTEN expression in gastric carcinomas. Tumori 91: 513-521, 2005.

14. Ding Y, Shimada Y, Kano M, Itami A, Kawabe A, Maeda M, Li Z, Hong T, Sato F, Kaganoi J, et al: PTEN/MMAC1 expression in esophageal squamous cell carcinomas. Int J Oncol 17: 695-699, 2000 .

15. Tachibana M, Shibakita M, Ohno S, Kinugasa S, Yoshimura H, Ueda S, Fujii T, Rahman MA, Dhar DK and Nagasue N: Expression and prognostic significance of PTEN product protein in patients with esophageal squamous cell carcinoma. Cancer 94: 1955-1960, 2002.

16. Chang D, Wang TY, Li HC, Wei JC and Song JX: Prognostic significance of PTEN expression in esophageal squamous cel carcinoma from Linzhou City, a high incidence area of northern China. Dis Esophagus 20: 491-496, 2007.

17. Damiano V, Rosa R, Formisano L, Nappi L, Gelardi T, Marciano R, Cozzolino I, Troncone G, Agrawal S, Veneziani BM, et al: Toll-like receptor 9 agonist IMO cooperates with everolimus in renal cell carcinoma by interfering with tumour growth and angiogenesis. Br J Cancer 108: 1616-1623, 2013.

18. Altorki N, Kent M, Ferrara C and Port J: Three-field lymph node dissection for squamous cell and adenocarcinoma of the esophagus. Ann Surg 236: 177-183, 2002.

19. Dresner SM and Griffin SM: Pattern of recurrence following radical oesophagectomy with two-field lymphadenectomy. Br J Surg 87: 1426-1433, 2000.
20. Thomas M, Greil J and Heidenreich O: Targeting leukemic fusion proteins with small interfering RNAs: Recent advances and therapeutic potentials. Acta Pharmacol Sin 27: 273-281, 2006.

21. Abbas-Terki T, Blanco-Bose W, Déglon N, Pralong W and Aebischer P: Lentiviral-mediated RNA interference. Hum Gene Ther 13: 2197-2201, 2002.

22. Fish RJ and Kruithof EK: Short-term cytotoxic effects and long-term instability of RNAi delivered using lentiviral vectors. BMC Mol Biol 5: 9, 2004.

23. Akhtar J, Wang Z, Zhang ZP and Bi MM: Lentiviral-mediated RNA interference targeting stathmin 1 gene in human gastric cancer cells inhibits proliferation in vitro and tumor growth in vivo. J Transl Med 11: 212, 2013.

24. Zhang C, Chakravarty D, Sakabe I, Mewani RR, Boudreau HE, Kumar D, Ahmad I and Kasid UN: Role of SCC-S2 in experimental metastasis and modulation of VEGFR-2, MMP-1, and MMP-9 expression. Mol Ther 13: 947-955, 2006.

25. Sawai H, Yasuda A, Ochi N, Ma J, Matsuo Y, Wakasugi T, Takahashi H, Funahashi H, Sato M and Takeyama H: Loss of PTEN expression is associated with colorectal cancer liver metastasis and poor patient survival. BMC Gastroenterol 8: 56 , 2008.

26. Chowdhury S, Ongchin M, Wan G, Sharratt E, Brattain MG and Rajput A: Restoration of PTEN activity decreases metastases in an orthotopic model of colon cancer. J Surg Res 184: 755-760, 2013.

27. Wikman H, Lamszus K, Detels N, Uslar L, Wrage M, Benner C, Hohensee I, Ylstra B, Eylmann K, Zapatka M, et al: Relevance of PTEN loss in brain metastasis formation in breast cancer patients. Breast Cancer Res 14: R49, 2012.

28. Tamura M, Gu J, Matsumoto K, Aota S, Parsons R and Yamada KM: Inhibition of cell migration, spreading, and focal adhesions by tumor suppressor PTEN. Science 280: 1614-1617, 1998.

29. Zhang LL, Liu J, Lei S, Zhang J, Zhou W and Yu HG: PTEN inhibits the invasion and metastasis of gastric cancer via downregulation of FAK expression. Cell Signal 26: 1011-1020, 2014.

30. Hu Y, Xu S, Jin W, Yi Q and Wei W: Effect of the PTEN gene on adhesion, invasion and metastasis of osteosarcoma cells. Oncol Rep 32: 1741-1747, 2014. 